\title{
Dual trigger with gonadotropin-releasing hormone agonist and recombinant human chorionic gonadotropin improves the outcome of intrauterine insemination
}

\author{
Binarwan Halim, PhD, Hilma Putri Lubis, MD \\ Department of Obstetrics and Gynecology, Faculty of Medicine, Universitas Sumatera Utara, Medan, Indonesia
}

\section{Objective}

The objective of this study was to evaluate the effectiveness of dual trigger, which is a combination of gonadotropinreleasing agonist ( $\mathrm{GnRH}-\mathrm{a}$ ) and recombinant human chorionic gonadotropin ( $\mathrm{hCG}$ ) in the final oocyte maturation, in the outcome of intrauterine insemination (IUI).

\section{Methods}

This retrospective observational study was conducted from January 2016 to October 2018 and involved 639 IUI cycles at the Halim Fertility Center, Indonesia. Controlled ovarian stimulation was performed during IUI cycles. The ovulation triggers were divided into two groups: group I received a combination of GnRH-a and recombinant hCG as a dual trigger, and group II received only recombinant hCG as a single trigger. The baseline characteristics, cycle parameters, and IUI outcomes of both groups were compared.

\section{Results}

Our study included a total of 639 IUI cycles, 334 were in the dual trigger group and 305 in the single trigger group. The clinical pregnancy rates were significantly higher in the dual trigger group than in the single trigger group $(P<0.001)$. Based on the multivariate analysis, the dual trigger increased the clinical pregnancy rate by 2.524 times than that by the single trigger.

\section{Conclusion}

Our data showed that the dual trigger combination of GnRH-a and recombinant hCG significantly improves the outcome of intrauterine insemination.

Keywords: IUI; Dual trigger; Single trigger; Outcome

\section{Introduction}

Intrauterine insemination (IUI) is the first treatment option for unexplained infertility after the failure of expectant treatment and before in vitro fertilization (IVF) [1]. It has been accepted as a more economical method than other assisted reproductive techniques. IUI is indicated for the treatment of unexplained infertility, mild male factor, cervical factor, anovulation, and minimal to mild endometriosis [1,2]. Success rates of IUI range between $10 \%$ and $20 \%$ per treatment $[1,3]$. Various methods are performed to increase the success rates of IUI, including ovarian stimulation, sperm preparation, endometrial preparation, ovulation trigger, increasing progesterone level, and monitoring of follicles [1]. However,
Received: 2021.08.26. Revised: 2021.12.15. Accepted: 2022.01.10. Corresponding author: Hilma Putri Lubis, MD

Department of Obstetrics and Gynecology, Faculty of Medicine, Universitas Sumatera Utara, Kampus Padang Bulan, Jalan Dr. Mansyur No.5, Medan 20155, Indonesia Email: hilmaputrilubis@gmail.com https://orcid.org/0000-0001-6752-2389

Articles published in Obstet Gynecol Sci are open-access, distributed under the terms of the Creative Commons Attribution Non-Commercial License (http://creativecommons. org/licenses/by-nc/3.0/) which permits unrestricted non-commercial use, distribution, and reproduction in any medium, provided the original work is properly cited.

Copyright $\odot 2022$ Korean Society of Obstetrics and Gynecology 


\section{Obstetrics \& Gynecology Science}

Vol. 65, No. 2, 2022

some factors that influence the success rate of IUI outcomes include the quality of oocytes and the appropriate timing of IUI.

Several studies have found that ovulation triggers affect the oocyte quality and the appropriate timing of IUI [1,4-6]. Ovulation is assumed to occur 36 to 38 hours after human chorionic gonadotropin [hCG] administration because of biochemical similarities between hCG and luteinizing hormone (LH) in ovulation induction. Andersen et al. [7] stated that the interval between $\mathrm{hCG}$ administration and ovulation was 38.3 hours (range, 34 to 46 hours). The number of patients in whom follicular rupture occurred more than 38.3 hours after hCG administration was 11 patients (34.3\%) [7]. As an alternative to hCG, the concept of a "dual trigger" that combines a bolus of gonadotropin-releasing agonist ( $\mathrm{GnRH}$ a) with $\mathrm{hCG}$ at the time of triggering has been investigated for IVF [8-15]. GnRH-a triggers have some benefits, such as prevention of ovarian hyperstimulation syndrome (OHSS), induction of follicle-stimulating hormone (FSH) surge, and individualized luteal phase support based on ovarian response to stimulation. FSH surge increases oocyte nuclear maturation, resumption of meiosis, and expansion of cumulus [16-20]. Zhang et al. [12] reported that the oocyte retrieval rate and the oocyte maturation rate in poor ovarian responders significantly increased in the dual trigger groups in a study of 1,389 cycles. Fabris et al. [10] observed that dual triggers increased the number of mature oocytes and oocyte maturation rates in women with a high immature oocyte rate in a previous cycle in a study of 163 ovarian stimulation cycles. Lin et al. [8] observed that the number of mature oocytes, implantation rates, clinical pregnancies, and live birth rates significantly increased in the dual trigger groups of normal responders in a study of 378 cycles of IVF. From the studies mentioned, an increased number of metaphase II (MII) oocytes in dual trigger maturation might be due to the more appropriate timing of oocyte retrieval and the combined effect of endogenous LH surge from GnRH trigger and exogenous LH effect from hCG $[8,10,12]$.

Similar to previous studies on dual triggers in IVF, we aimed to evaluate the outcome of the dual trigger in the IUI procedure. The objective of this study was to evaluate the effectiveness of the dual trigger, which is combined of GnRH$a$ and recombinant $\mathrm{hCG}$ for final oocyte maturation in the outcome of IUI.

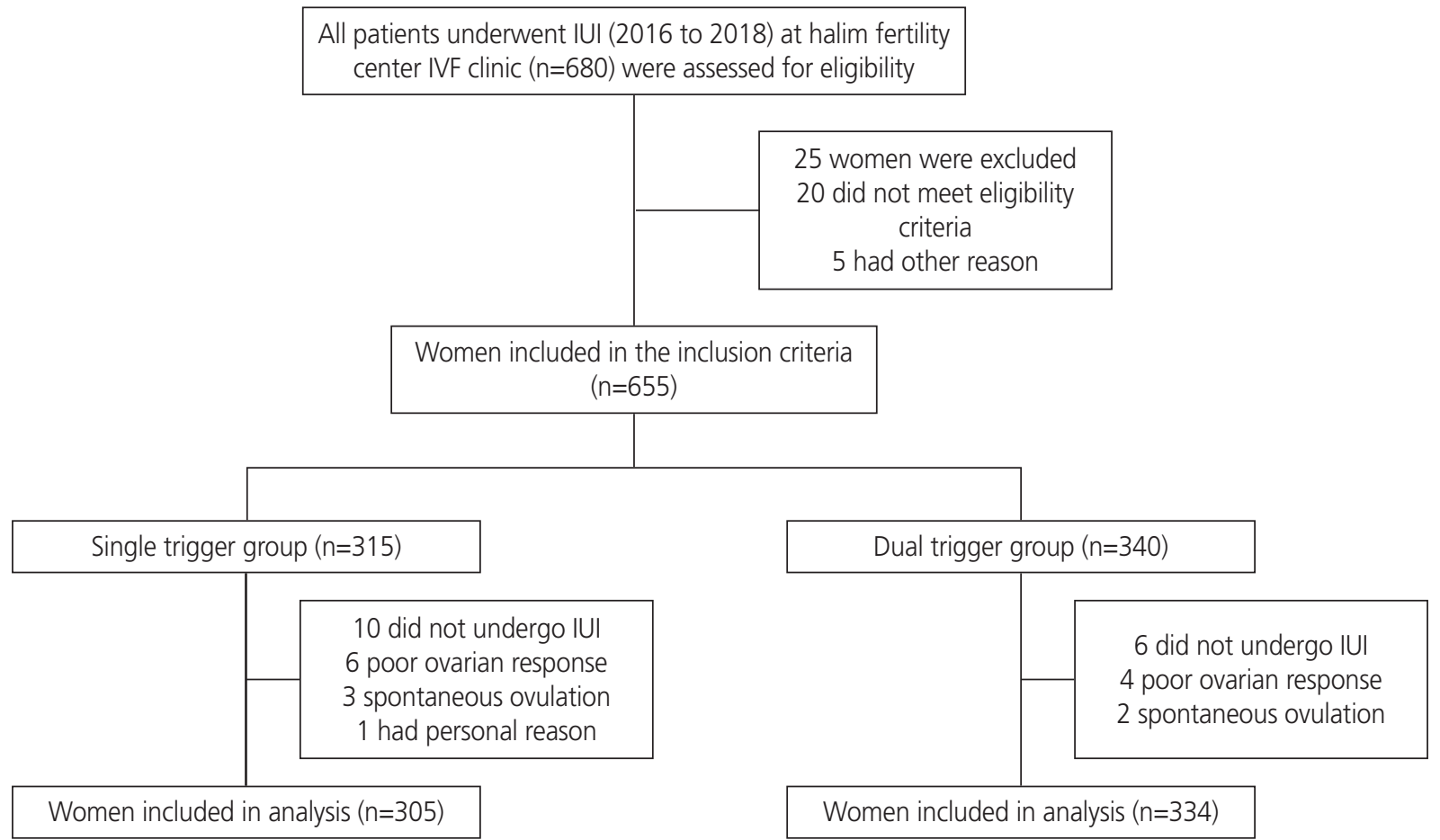

Fig. 1. Schematic study of single trigger and dual trigger in IUI. IUI, intrauterine insemination; IVF, in vitro fertilization. 


\section{Obstetrics \& Gynecology Science}

Binarwan Halim, et al. Dual trigger improves the outcome of IUI

\section{Material and methods}

\section{Study design and participants}

This retrospective observational study was conducted at the Halim Fertility Center IVF Center between January 2016 and October 2018. Couples seeking infertility treatment were eligible if they fulfilled one of the following inclusion criteria: female aged $<40$ years, unexplained infertility, bilaterally patent tubes, mild male factor with total motile sperm count (TMSC) $10-20 \times 10^{6} / \mathrm{mL}$ ), FSH level $<10 \mathrm{mlU} / \mathrm{mL}$, and anovulation. Exclusion criteria were pre-existing medical conditions, female aged $>40$ years, endometriosis cyst, adenomyosis, congenital uterine anomalies, severe male infertility with TMSC $\leq 5 \times 10^{6}$, spontaneous ovulation, and poor ovarian response. We used single trigger and dual trigger in this study based on the period of time. The single trigger was used for final oocyte maturation from January 2016 to May 2017, and the dual trigger was used from June 2017 to October 2018 (Fig. 1).

\section{IUI protocol}

We stimulated our patients with gonadotropins on day 2 or 3 of the cycle using $75 \mathrm{IU}$ of recombinant FSH (Gonal-F, Merck KGaA, Germany). Ovarian stimulation was monitored using ultrasound to measure the follicle diameter and endome- trial growth from day 8 of the cycle until the follicle reached a diameter of $\geq 181 \mathrm{~mm}$. When the leading follicle reached $18 \mathrm{~mm}$ in diameter, we observed the final oocyte maturation. In the single trigger group, it was triggered by only recombinant hCG (Ovidrel $250 \mu$ g; Merck KGaA, Darmstadt, Germany) and in the dual trigger group, ovulation was triggered with GnRH-a (Lucrin 1 mg; AbbVie, New South Wales, Australia) and recombinant hCG (Ovidrel $250 \mu \mathrm{g}$, Merck $\mathrm{KgaA}$ ) at the same time. The IUI procedure was performed only once, 36 hours after ovulation triggering.

\section{Semen preparation and insemination}

Semen samples were collected at the Halim fertility center after 3 to 5 days of sexual abstinence. Fresh sperm was used for the IUI. The swim-up protocol was used for all the samples. Insemination was performed 36 hours after triggering with either single or dual trigger.

\section{Luteal phase support}

We supported the luteal phase with oral micronized progesterone $300 \mathrm{mg}$ for 14 days. The biochemical pregnancy rate was observed after 14 days using a urinary pregnancy test. Clinical pregnancy was defined by the presence of fetal cardiac activity and an intrauterine gestational sac confirmed by transvaginal ultrasound 2 weeks after a positive urinary pregnancy test.

Table 1. The participants' demographic data

\begin{tabular}{lccc}
\hline Variable & Single trigger group $(\mathbf{n = 3 0 5})$ & Dual trigger group (n=334) & $\boldsymbol{P}$-value \\
\hline Female age (yr) & $32(22-40)$ & $32(21-40)$ & $0.945^{\mathrm{a})}$ \\
Infertility duration (yr) & $4(1-10)$ & $4(2-13)$ & $0.048^{\mathrm{a})}$ \\
Etiology of Infertility & $111(36.6)$ & $126(37.7)$ & $0.841^{\mathrm{b})}$ \\
Mild male factor (\%) & $88(29)$ & $90(26.9)$ & $118(35.3)$ \\
Female factor (\%) & $106(34.3)$ & $6.5(3.6-9.7)$ & $0.411^{\mathrm{a})}$ \\
Combined (\%) & $6.3(3.2-9.7)$ & $2.4(1.1-5.2)$ & $0.340^{\mathrm{a})}$ \\
FSH (mIU/mL) & $2.7(1.1-5.2)$ & $900(750-975)$ & $0.873^{\mathrm{a})}$ \\
AMH (ng/mL) & $900(750-975)$ & $17.3(14.2-20.9)$ & $0.488^{\mathrm{a})}$ \\
Total amount of rFSH (IU) & $17.2(14.2-20.9)$ & $35(29-46)$ & $0.847^{\mathrm{a})}$ \\
Sperm concentration 106/mL & $35(29-46)$ & $5(2-9)$ & $0.916^{\mathrm{a})}$ \\
Sperm motility (A+B\%) & $5(2-9)$ & $12.6(7.9-17.2)$ & $0.545^{\mathrm{a})}$
\end{tabular}

FSH, follicle-stimulating hormone; $\mathrm{AMH}$, antimullerian hormone; $r \mathrm{FSH}$, recombinant follicle stimulating hormone; TMSC, total motile sperm count.

${ }^{\text {a) } M a n n ~ w h i t n e y ; ~}{ }^{\text {b) }}$ Chi square. 


\title{
Obstetrics \& Gynecology Science
}

\author{
Vol. 65, No. 2, 2022
}

\section{Statistical analysis}

We used the Kolmogorov-Smirnov test to assess the normality of the sample quantitative variables. The data were nonparametric, and baseline comparisons were made using the Mann-Whitney $U$ test. Mann-Whitney and chi-squared tests were used to analyze demographic characteristics. The Mann-Whitney $U$ test was also used to analyze endometrial thickness, the number of basal antral follicles, and the number of follicles $>17 \mathrm{~mm}$. Furthermore, we used the chisquared test to analyze clinical pregnancy rates, multiple pregnancies, and OHSS rates between the two groups. A multivariate logistic regression analysis was used to evaluate factors related to the outcome of IUI. Analyses were performed using the Statistical Package for Social Sciences version 20.0 (SPSS, Chicago, IL, USA). Statistical significance was set at $P<0.05$.

\section{Results}

From January 2016 to October 2018, 639 IUI cycles were included in this study. Table 1 presents the characteristics of participants: the median age of participants in the dual trigger group was 32 years (range, 21 to 40 years), and there was no significant difference in the ages of women between the single trigger group and the dual trigger group $(P=0.945)$. The median duration of infertility in the dual trigger group was 4 years (range, 2 to 13 years), and there was a signifi- cant difference in the duration of infertility between the two groups $(P=0.048)$. In this study, the etiology of infertility in single and dual trigger groups was mild male factor, but there was no significant difference between them $(P=0.841)$. The median basal FSH level in the dual trigger group was 6.5 with no significant difference in basal FSH levels between the two groups $(P=0.411)$. The median antimullerian hormone $(\mathrm{AMH})$ in the dual trigger was 2.4 with no significant difference in the AMH level between the two groups $(P=0.340)$ [20]. The median total amount of rFSH in the dual trigger group was similar to that of the single trigger group, and there was no significant difference in the total amount of rFSH between the two groups $(P=0.873)$. The median sperm concentration, motility, and morphology were similar between the two groups with no significant difference ( $P>0.05)$. The median TMSC in the dual trigger group was 12.6 and there was no significant difference in the number of TMSCS between the single trigger group and the dual trigger group ( $P=0.545)$.

As shown in Table 2, the median endometrial thickness in the dual trigger group was similar to that in the single trigger group (range, 5-16 mm) with no significant difference in endometrial thickness between the two groups $(P=0.337)$. The median number of basal antral follicles in the dual trigger group was seven follicles (range, 1 to 34 follicles), but there was no significant difference in basal antral follicle count between the two groups ( $P=0.587)$. The median number of follicles $>17 \mathrm{~mm}$ in the single and dual trigger groups was two

Table 2. The stimulation cycle parameters

\begin{tabular}{lccc}
\hline Parameter & Single trigger group $(\mathbf{n = 3 0 5})$ & Dual trigger group $(\mathbf{n}=334)$ & $\boldsymbol{P}$-value \\
\hline Endometrial thickness $(\mathrm{mm})$ & $9(5-19)$ & $9(5-16)$ & $0.337^{\mathrm{a})}$ \\
Number of basal antral follicles & $7(1-41)$ & $7(1-34)$ & $0.587^{\mathrm{a})}$ \\
Number of follicles $>17 \mathrm{~mm}$ & $2(1-3)$ & $2(1-3)$ & $0.974^{\mathrm{a})}$ \\
\hline
\end{tabular}

a) Mann whitney

Table 3. Outcome of the intrauterine insemination procedure

\begin{tabular}{lccc}
\hline Parameter & Single trigger group $(\mathbf{n = 3 0 5})$ & Dual trigger group $(\mathbf{n = 3 3 4 )}$ & $\boldsymbol{P}$-value \\
\hline Clinical pregnancy rate & $43 / 305(14.1)$ & $96 / 334(28.7)$ & $<0.001^{\mathrm{a})}$ \\
Multiple pregnancy rate & $3 / 43(7.0)$ & $7 / 96(7.3)$ & $0.627^{\mathrm{a})}$ \\
OHSS & $8 / 305(2.6)$ & $9 / 334(2.7)$ & $0.576^{\mathrm{a})}$ \\
\hline
\end{tabular}

Values are presented as number (\%).

OHSS, Ovarian Hyperstimulation Syndrome.

${ }^{\text {a) }}$ Chi square. 


\section{Obstetrics \& Gynecology Science}

Binarwan Halim, et al. Dual trigger improves the outcome of IUI

follicles (range, 1 to 3 follicles), and there was no significant difference in the number of follicles $>17 \mathrm{~mm}$ between the two groups $(P=0.974)$.

In Table 3, we observe that the clinical pregnancy rate was higher in the dual trigger group than in the single trigger group ( $28.7 \%$ vs $14.1 \%)$, and there was a significant difference between the two groups $(P<0.001)$. Although the multiple pregnancy rates were higher in the dual trigger group than in the single trigger group $(7.3 \%$ vs. $7 \%)$, there was no significant difference between the groups $(P=0.627)$. Furthermore, we found that the OHSS rate was not significantly higher in the dual trigger group than in the single trigger group $(2.7 \%$ vs. $2.6 \%)$, but there was no significant difference between the two groups ( $P=0.576)$. Most of OHSS in the dual trigger group was in the mild form.

As shown in Table 4, after the seventh selection of variables related to the outcome of IUI, which consisted of female age, infertility duration, etiology of infertility, FSH and AMH levels, total amount of $\mathrm{rFSH}$, sperm concentration, sperm motility, sperm morphology, TMSC, endometrial thickness, number of basal antral follicles, number of follicles $>17 \mathrm{~mm}$, and dual trigger, there were some factors that were related to the outcome of IUI: female age, endometrial thickness, and dual trigger. Based on the relative risk (RR) in this study, it can be concluded that the majority of the successful outcomes of IUI was influenced by a dual trigger with an RR value of 2.524 (95\% Cl, 1.681-3.790), which means that dual trigger could increase the clinical pregnancy rate by 2.524 times than that by the single trigger.

\section{Discussion}

In this study, we found that the clinical pregnancy rate was significantly higher in the dual trigger group than in the sin- gle trigger group (14.1\% vs. $28.7 \% ; P<0.001)$. Based on the multivariate analysis, the dual trigger could increase the clinical pregnancy rate by 2.524 times than that by single trigger. This result was similar to that of other studies on IVF.

Final oocyte maturation with human chorionic gonadotropin ( $h C G$ ) is commonly performed in patients undergoing IUI. However, in assisted reproductive cycles, hCG triggers are associated with a higher risk of developing OHSS and premature LH surge. Other than HCG, GnRH-a has been used as an alternative to induce ovulation in the last two decades. GnRH agonists induce the endogenous surge of LH and FSH by their flare-up effect, which is helpful in triggering ovulation and decreasing the risk of OHSS [1-3]. Ovulation triggers with hCG or GnRH are widely used in the IUI cycle.

Ding et al. [15] observed that implantation and pregnancy rates were higher in the dual trigger group than in the hCGonly trigger group. In addition, Chen et al. [14] reported that the dual trigger group had significantly higher clinical pregnancy rates than those of the hCG-only group. The dual trigger of final oocyte maturation with GnRH-a and hCG improves the outcomes of IVF in patients with normal responders, poor ovarian responders, high proportion of immature oocytes, and high responders [8,9,12,13,21-23]. These studies reveal that the final maturation of oocyte with dual trigger improves the number of mature oocytes (MII) and oocyte quality, which is reflected by the higher number of good quality embryos, thus improving the clinical pregnancy rate. HCG has traditionally been used as the gold standard for triggering oocyte maturation in controlled ovarian stimulation cycles [1-3,24]. GnRH-a is one of the triggers of final oocyte maturation that is used as an alternative to hCG to prevent OHSS in PCOS patients [16-19]. GnRH-a can stimulate FSH surge and LH surge. FSH surge has a beneficial effect on oocyte maturation and causes cumulus expansion of the oocyte [16-19,21,23].

Table 4. Multivariate analysis of factors related to the outcome of IUI

\begin{tabular}{|c|c|c|c|c|c|}
\hline \multirow{2}{*}{ Variable } & \multicolumn{2}{|c|}{$95 \% \mathrm{Cl}$ for $\mathrm{RR}$} & \multirow{2}{*}{ B } & \multirow{2}{*}{ RR } & \multirow{2}{*}{$P$-value } \\
\hline & Lower & Upper & & & \\
\hline Female age & 0.888 & 0.980 & -0.069 & 0.933 & 0.006 \\
\hline Endometrial thickness & 1.032 & 1.246 & 0.126 & 1.134 & 0.009 \\
\hline Dual trigger & 1.681 & 3.790 & 0.926 & 2.524 & 0.000 \\
\hline Constant & & & -0.801 & 0.449 & 0.409 \\
\hline
\end{tabular}

IUI, intrauterine insemination; Cl, confidence interval; RR, relative risk; B, regression coefficient. 


\section{Obstetrics \& Gynecology Science}

Vol. 65, No. 2, 2022

This study also showed that in addition to increasing the number of oocyte maturation and oocyte quality, the purpose of triggering ovulation with the dual trigger in IUI is also related to the appropriate timing of IUI. The timing of IUI should be as close as possible to the time of ovulation to increase the success rate of IUI. Early studies reported discrepancies in the time of ovulation after an hCG trigger. Ovulation was expected 36 hours after hCG administration. Andersen et al. [7] found that follicular rupture occurred more than 38.3 hours after hCG administration in $34.3 \%$ of patients. AboulGheit [25] found that the clinical pregnancy rate per cycle was higher in the group in which ovulation occurred 48 hours after hCG administration than that in the 34 -hours group and 24 -hours group (19\% vs. $14.6 \%$ vs. $9.5 \%$, respectively). The above study observed that partial ovulation occurred at 36 hours after hCG-only trigger and some ovulation occurred after more than 36 hours. The final maturation oocyte with the dual trigger is expected to be even closer to the time of ovulation at 36 hours so that IUI could be performed closer to the time of ovulation (36 hours).

The main limitation of this study was its retrospective nature, as data sampling was not well-controlled. Data recruitment was based on a period because we modified the ovulation trigger from a single trigger to a dual trigger after approximately 1 year, and then we evaluated the outcome of IUI between the single and dual trigger groups.

Further prospective studies are needed to validate the results of this study and avoid bias. A prospective study should be performed in a randomized controlled trial using a homogeneous sample, for instance, in an unexplained infertility group.

To the best of our knowledge, this is the first study to evaluate the outcome of the dual trigger on ovulation triggers in IUI.

In conclusion, ovulation with dual triggers significantly improve the outcome of IUI. Further well-designed prospective studies are required to validate these results.

\section{Conflict of interest}

No potential conflict of interest relevant to this article was reported.

\section{Ethical approval}

This study was approved by the Health Research Ethical Committee of Stella Maris Women's and Children's Hospital on October 20, 2018 (reference number :1496-1/Dir/RSIA). SM/X/2018).

\section{Patient consent}

In this study, informed consent was obtained from all the participants.

\section{Funding information}

None.

\section{Acknowledgments}

The authors thank the Halim Fertility Center and the team of embryologists for their help and support of this study.

\section{References}

1. Soliman BS, Siam S. Pregnancy rate after ovulation triggering with gonadotrophin releasing hormone agonist versus human chorionic gonadotrophin in women undergoing controlled ovarian stimulation/intrauterine insemination. Middle East Fertil Soc J 2014;19:262-7.

2. Weiss A, Beck-Fruchter R, Lavee M, Geslevich Y, Golan J, Ermoshkin $A$, et al. A randomized trial comparing time intervals from HCG trigger to intrauterine insemination for cycles utilizing GnRH antagonists. Syst Biol Reprod Med 2015;61:44-9.

3. Le MT, Nguyen DN, Zolton J, Nguyen VQH, Truong QV, Cao NT, et al. GnRH agonist versus hCG trigger in ovulation induction with intrauterine insemination: a randomized controlled trial. Int J Endocrinol 2019 Mar 13 [Epub]. https://doi.org/10.1155/2019/2487067.

4. Farag AH, El-Deen MHN, Hassan RM. Triggering ovulation with gonadotropin-releasing hormone agonist versus human chorionic gonadotropin in polycystic ovarian 


\section{Obstetrics \& Gynecology Science}

Binarwan Halim, et al. Dual trigger improves the outcome of IUI

syndrome. A randomized trial. Middle East Fertil Soc J 2015;20:217-23.

5. Lee Jl, Hur YM, Jeon ES, Yoon JI, Jung GS, Hong KE, et al. Comparison of pregnancy rates by intrauterine insemination after ovulation trigger with endogenous $\mathrm{LH}$ surge, GnRH agonist or hCG in stimulated cycles. Clin Exp Reprod Med 1999;26:389-98.

6. Taheripanah R, Zamaniyan M, Moridi A, Taheripanah A, Malih N. Comparing the effect of gonadotropin-releasing hormone agonist and human chorionic gonadotropin on final oocytes for ovulation triggering among infertile women undergoing intrauterine insemination: An RCT. Int J Reprod Biomed 2017;15:351-6.

7. Andersen AG, Als-nielsen B, Hornnes PJ, Andersen LF. Time interval from human chorionic gonadotrophin (HCG) injection to follicular rupture. Hum Reprod 1995;10:3202-5.

8. Lin MH, Wu FS, Lee RK, Li SH, Lin SY, Hwu YM. Dual trigger with combination of gonadotropin-releasing hormone agonist and human chorionic gonadotropin significantly improves the live-birth rate for normal responders in GnRH-antagonist cycles. Fertil Steril 2013;100:1296-302.

9. Griffin D, Feinn R, Engmann L, Nulsen J, Budinetz T, Benadiva C. Dual trigger with gonadotropin-releasing hormone agonist and standard dose human chorionic gonadotropin to improve oocyte maturity rates. Fertil Steril 2014;102:405-9.

10. Fabris AM, Cruz M, Legidos V, Iglesias C, Muñoz M, García-Velasco JA. Dual triggering with gonadotropinreleasing hormone agonist and standard dose human chorionic gonadotropin in patients with a high immature oocyte rate. Reprod Sci 2017;24:1221-5.

11. Mahajan N, Sharma S, Arora PR, Gupta S, Rani K, Naidu $P$. Evaluation of dual trigger with gonadotropin-releasing hormone agonist and human chorionic gonadotropin in improving oocyte maturity rates: a prospective randomized study. J Hum Reprod Sci 2016;9:101-6.

12. Zhang J, Wang Y, Mao X, Chen Q, Hong Q, Cai R, et al. Dual trigger of final oocyte maturation in poor ovarian responders undergoing IVF/ICSI cycles. Reprod Biomed Online 2017;35:701-7.

13. Li S, Zhou D, Yin T, Xu W, Xie Q, Cheng D, et al. Dual trigger of triptorelin and HCG optimizes clinical outcome for high ovarian responder in GnRH-antagonist protocols. Oncotarget 2018;9:5337-43.
14. Chen $\mathrm{CH}$, Tzeng CR, Wang PH, Liu WM, Chang HY, Chen $\mathrm{HH}$, et al. Dual triggering with $\mathrm{GnRH}$ agonist plus hCG versus triggering with hCG alone for IVF/ICSI outcome in GnRH antagonist cycles: a systematic review and meta-analysis. Arch Gynecol Obstet 2018;298:17-26.

15. Ding N, Liu X, Jian Q, Liang Z, Wang F. Dual trigger of final oocyte maturation with a combination of $\mathrm{GnRH}$ agonist and hCG versus a hCG alone trigger in $\mathrm{GnRH}$ antagonist cycle for in vitro fertilization: a systematic review and meta-analysis. Eur J Obstet Gynecol Reprod Biol 2017;218:92-8.

16. Kol S, Humaidan P. GnRH agonist triggering: recent developments. Reprod Biomed Online 2013;26:226-30.

17. Youssef MA, Van der Veen F, Al-Inany HG, Mochtar MH, Griesinger G, Nagi Mohesen M, et al. Gonadotropinreleasing hormone agonist versus HCG for oocyte triggering in antagonist-assisted reproductive technology. Cochrane Database Syst Rev 2014;(10):CD008046.

18. Orvieto R. Triggering final follicular maturation- hCG, GnRH-agonist or both, when and to whom? J Ovarian Res 2015;8:60.

19. Humaidan $P$, Bredkjaer $H E$, Bungum $L$, Bungum $M$, Grøndahl ML, Westergaard L, et al. GnRH agonist (buserelin) or hCG for ovulation induction in GnRH antagonist IVF/ICSI cycles: a prospective randomized study. Hum Reprod 2005;20:1213-20.

20. Scantamburlo VM, Linsingen RV, Centa LJR, Toso KFD, Scaraboto D, Araujo Júnior $E$, et al. Association between decreased ovarian reserve and poor oocyte quality. Obstet Gynecol Sci 2021;64:532-9.

21. Haas J, Zilberberg E, Dar S, Kedem A, Machtinger R, Orvieto R. Co-administration of GnRH-agonist and hCG for final oocyte maturation (double trigger) in patients with low number of oocytes retrieved per number of preovulatory follicles--a preliminary report. J Ovarian Res 2014;7:77

22. Zilberberg E, Haas J, Dar S, Kedem A, Machtinger R, Orvieto R. Co-administration of GnRH-agonist and hCG, for final oocyte maturation (double trigger), in patients with low proportion of mature oocytes. Gynecol Endocrinol 2015;31:145-7.

23. Alyasin A, Mehdinejadiani S, Ghasemi M. GnRH agonist trigger versus hCG trigger in $\mathrm{GnRH}$ antagonist in IVF/ICSI cycles: a review article. Int J Reprod Biomed 2016;14:557-66. 


\section{Obstetrics \& Gynecology Science}

Vol. 65, No. 2, 2022

24. Conghui Liu, Yu Li, Hong Jiang, Yingchun Liu, Xiaomin Song. The clinical outcomes of fresh versus frozen embryos transfer in women $\geq 40$ years with poor ovarian response. Obstet Gynecol Sci 2021;64:284-92.
25. AboulGheit S. Pregnancy rates following three different timings of intrauterine insemination for women with unexplained infertility: a randomised controlled trial. Middle East Fertil Soc J 2010;15:265-8. 